\title{
Chemical Engineering of Molecular Qubits
}

\author{
C. J. Wedge, ${ }^{1}$ G. A. Timco, ${ }^{2}$ E. T. Spielberg, ${ }^{1}$ R. E. George, ${ }^{1}$ F. Tuna, ${ }^{2}$ S. Rigby, ${ }^{3}$ E. J. L. McInnes,${ }^{2}$ \\ R. E. P. Winpenny, ${ }^{2}$ S. J. Blundell, ${ }^{1}$ and A. Ardavan ${ }^{1, *}$ \\ ${ }^{1}$ Centre for Advanced Electron Spin Resonance, Clarendon Laboratory, Department of Physics, \\ University of Oxford, OXI 3PU, United Kingdom \\ ${ }^{2}$ School of Chemistry and Photon Science Institute, University of Manchester, Oxford Road, Manchester, M13 9PL, United Kingdom \\ ${ }^{3}$ Manchester Interdisciplinary Biocentre, University of Manchester, Oxford Road, Manchester, M13 9PL, United Kingdom
}

(Received 29 September 2011; published 7 March 2012)

\begin{abstract}
We show that the electron spin phase memory time, the most important property of a molecular nanomagnet from the perspective of quantum information processing, can be improved dramatically by chemically engineering the molecular structure to optimize the environment of the spin. We vary systematically each structural component of the class of antiferromagnetic $\mathrm{Cr}_{7} \mathrm{Ni}$ rings to identify the sources of decoherence. The optimal structure exhibits a phase memory time exceeding $15 \mu$ s.
\end{abstract}

DOI: 10.1103/PhysRevLett.108.107204

Molecular magnets exhibit a range of properties that are useful for the components of a quantum computer: the electron spin structures are flexible and tunable [1]; high spin molecules offer dense quantum memories and can host elementary quantum algorithms [2,3]; superstructures incorporating multiple coupled molecular magnets can be self-assembled by chemical means [4-6]; and they can be deposited onto gold and graphite surfaces suitable for interfacing with top-down nanoscale devices for single molecule measurements [7,8]. Antiferromagnetically coupled spin clusters can form well-defined qubits, inheriting the long coherence times of their constituent spins, and perturbations effecting single and two-qubit gates may be applied on the molecular length scale rather than on the single-spin length scale $[9,10]$. The controllable excitations exhibited by well-defined spin structures represent a resource for single- and multiqubit manipulations that is not available for single spins [11].

In a $\mathrm{Cr}_{7} \mathrm{Ni}$ molecular nanomagnet $\left(S=\frac{1}{2}\right.$ ground state) coherence times exceed by a large factor the time needed for single-qubit manipulations [12]. By studying the effect on the spin dynamics of varying each structural component of the nanomagnet, we show in this Letter that the conformational rigidity of the molecule is important as well as simply the number of spin active nuclei present, and that the dominant contributions to electron spin decoherence in this system are nuclear spin diffusion and spectral diffusion being driven by dynamic processes including methyl group rotation and libration.

We have investigated the influence on the decoherence time of varying the two key components of the $\mathrm{Cr}_{7} \mathrm{Ni}$ molecular magnet system, the central templating cation and the carboxylate bridging ligand (Fig. 1). The parent compound for this class of molecular magnets is the ring $\mathrm{Cr}_{8} \mathrm{~F}_{8} \mathrm{Piv}_{16}[13,14]$, which has a diamagnetic ground state. It consists of a ring of octahedrally coordinated trivalent chromium ions, each bridged to its neighbor by one
PACS numbers: 75.50.Xx, 03.67. $-\mathrm{a}, 33.35 .+\mathrm{r}, 76.30 .-\mathrm{v}$

fluoride and two bulky pivalate (2,2-dimethylpropanoate) ligands. Antiferromagnetic coupling between the eight $\mathrm{Cr}^{3+}\left(s=\frac{3}{2}\right)$ gives a ground state total spin $S=0$. However, by substituting one $\mathrm{Cr}^{3+}$ with a divalent metal ion, and templating around a suitable cation to balance the negative charge of the heterometallic ring, it is possible to produce compounds with paramagnetic ground states [15]. Here, we discuss exclusively compounds of the type $\mathrm{Cr}_{7} \mathrm{Ni}$, in which the inclusion of $\mathrm{Ni}^{2+}(s=1)$ leads to a ground state $\operatorname{spin} S=\frac{1}{2}$.

To determine the influence of dynamic effects, we studied phase-coherence times over a range of temperatures by observation of the decay of the primary echo generated by the standard two pulse echo sequence,
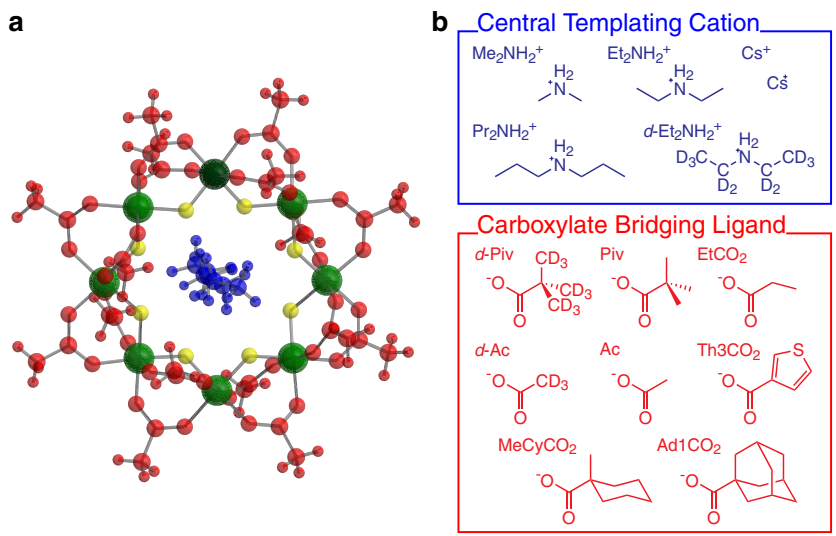

FIG. 1 (color online). Structures of the $\mathrm{Cr}_{7} \mathrm{Ni}$ molecules. (a) Crystal structure of $\left[{ }^{n} \mathrm{Pr}_{2} \mathrm{NH}_{2}\right]\left[\mathrm{Cr}_{7} \mathrm{NiF}_{8} \mathrm{Ac}_{16}\right]$. The colored balls represent either different atom types: $\mathrm{Cr}$ (light green), $\mathrm{Ni}$ (dark green), F (yellow), or different interchangeable substituents: central templating cation (blue), carboxylate bridging ligand (red). (b) Appropriately color coded chemical structures of some possible variants with abbreviated names. The abundance of hydrogen atoms in the molecular building blocks is tabulated in the Supplemental Material [34]. 
[ $\frac{\pi}{2}-\tau$ - $\pi$ - $\tau$-echo] [16]. In all cases at $T \gtrsim 8 \mathrm{~K}$ the spin echo is lost rapidly due to fast relaxation as the low-lying $S=\frac{3}{2}$ excited state becomes thermally populated $(\Delta E=13 \mathrm{~K}$ at zero field) $[17,18]$. For many of the samples that we studied the echo decay was not a simple monoexponential, so to determine the phase memory times we fit the decays with a stretched exponential function of the form

$$
Y(2 \tau)=Y(0) \exp \left(-\left(2 \tau / T_{m}\right)^{x}\right),
$$

where $T_{m}$ is the phase memory time, $x$ the stretch parameter, $Y(2 \tau)$ the echo intensity for a pulse separation $\tau$, and $Y(0)$ the intensity extrapolated to $\tau=0$. The shape of the echo decay curve is dependent upon both the dominant dephasing mechanism and the rate of this process [19].

In order to ensure that dipolar interactions between magnetic molecules do not contribute to dephasing, we diluted all samples such that the dephasing was independent of concentration. In this limit, interactions with environmental nuclear spins dominate via several possible mechanisms. First, in the rigid limit local magnetic field fluctuations at the electron are caused by nuclear spin flipflop processes, which, although conserving nuclear magnetization, modulate the electron-nuclear dipolar interaction hence driving irreversible dephasing of the electron spin. This mechanism is known as nuclear spin diffusion and, theoretically, gives rise to a stretch parameter $2 \leq x \leq$ 3 depending on details of the model [19-21]. Though the rate of individual nuclear spin flip-flops is low (typically $\sim 10 \mathrm{kHz}$ ), the large bath of coupled nuclear spins contributes to the efficiency of the process. Second, motion of the nuclear spins may lead to a temperature-dependent contribution to dephasing known as spectral diffusion. In the case of translational diffusion of protons relative to the electron spin, even at slow motional rates, this second contribution may become comparable to that from nuclear spin diffusion (indeed, the effects of the latter process may be averaged out by motion [22,23]). The onset of spectral diffusion is associated with a change in the shape of the echo decay, characterized by a reduction in both $T_{m}$ and the stretch parameter $x$.

Motional effects may arise not only from nuclei diffusing but also from internal rotations about bond axes leading to nuclear spin flips, which in turn drive spectral diffusion (vibrations will remain "frozen out" to much higher temperatures and need not be considered). Such effects have been particularly associated with rotation of methyl groups, but have also been seen for $180^{\circ}$ flips of phenyl rings $[19,24]$. Fast rotations make a negligible dephasing contribution, whereas slower motions are more effective in producing nuclear spin flips [25]. Studies of phase memory times have often been concerned with the temperature regime in which the activated free rotation of methyl groups is still possible, characterized by an increase in $T_{m}$ with increasing temperature. However, even at lower temperatures methyl groups are important in driving dephasing with less hindered groups having greater efficiency [26]. Barriers to methyl rotation are of the order $10^{2}-10^{3} \mathrm{~K}$ in glassy media, but even at temperatures where classical rotation is frozen out small angle librations can occur and, for $T \lesssim 25 \mathrm{~K}$, quantum tunneling rotations $[27,28]$ are possible.

Rotations of methyl groups are often considered semiclassically, but in the low temperature limit it is also necessary to consider the quantization of this rotation [29]. The form of the rotational potential depends on the environment of the methyl group. For example, in toluene, the methyl group has a $C_{3}$ axis, but being coincident with a $C_{2}$ axis of the planar phenyl ring this gives rise to a sixfold potential with maxima when a $\mathrm{C}-\mathrm{H}$ bond of the methyl group eclipses the $\mathrm{C}-\mathrm{C}$ bond of the ring [30]. If the symmetry of the ring were lower, both threefold and sixfold terms could become important. Although these factors complicate quantitative modelling, the librational modes are in any case approximately harmonic. In the limit of small displacements the eigenfrequency of oscillation $\nu \sim \sqrt{V / I}$, where $I$ is the moment of inertia, and $V$ characterizes the height of the potential, and the mean square displacement $\overline{\Delta \theta^{2}} \sim 1 / \sqrt{V I}$. Although deuteration might be expected to suppress tunneling rotations owing to the larger mass, it increases $I$, making librational excitations more accessible. Furthermore, the different exchange symmetries of $\mathrm{CH}_{3}$ and $\mathrm{CD}_{3}$ may affect the tunneling dynamics [29,31].

The fitting parameters for the various compounds at $5 \mathrm{~K}$ are tabulated in Table I. At this temperature there is an almost threefold variation in the phase memory times from $\sim 300 \mathrm{~ns}$ to over $900 \mathrm{~ns}$. There are two distinct groups of compounds: those for which $x$ is close to 1 (characteristic of spectral diffusion), and those for which $x>1.5$ (characteristic of nuclear spin diffusion). Compounds in which the protons are susceptible to positional disorder, i.e., those whose bridging ligands contain groups that can rotate, predominate in the latter group. This indicates that the disorder-induced broadening of the nuclear spectrum enhances the nuclear spin diffusion more than the possibility of rotation enhances spectral diffusion.

To illustrate this point we compare the temperature dependence of the relaxation parameters for three compounds, (labeled 1, 2, and $\mathbf{3}$ in Table I) in Fig. 2. For compound 1, which contains 48 methyl groups on the $\mathrm{Cr}_{7} \mathrm{Ni}$ ring, the stretch parameter increases as the temperature decreases, and correspondingly, $T_{m}$ plateaus at low temperature as the limiting value for nuclear spin diffusion is reached. Motion of the methyl groups, driving spectral diffusion, reduces both $T_{m}$ and $x$ at higher temperatures. For the perdeuterated analogue, compound 2, the decay is close to monoexponential around 5 K. $x$ rises on cooling, but more slowly than for the protonated isotopomer. Again, nuclear spin diffusion and spectral diffusion compete, but nuclear spin diffusion is less efficient. Owing to the lower 
TABLE I. Relaxation parameters for variously substituted $\mathrm{Cr}_{7} \mathrm{Ni}$ rings at $5 \mathrm{~K}$. Abbreviated chemical names are given in Fig. 1. The errors indicated are the uncertainty in fitting parameters for a single measurement.

\begin{tabular}{lccrc}
\hline \hline Label & Carboxylate bridging ligand & Central templating cation & $T_{m}(\mathrm{~ns})$ & $x$ \\
\hline & $\mathrm{Piv}$ & $\mathrm{Me}_{2} \mathrm{NH}_{2}^{+}$ & $378(3)$ & $1.12(1)$ \\
$\mathbf{1}$ & $\mathrm{Piv}$ & $\mathrm{Et}_{2} \mathrm{NH}_{2}^{+}$ & $725(1)$ & $1.73(1)$ \\
$\mathbf{4}$ & $\mathrm{Piv}$ & ${ }^{n} \mathrm{Pr}_{2} \mathrm{NH}_{2}^{+}$ & $622(1)$ & $1.60(1)$ \\
$\mathbf{1 b}$ & $\mathrm{Piv}$ & $\mathrm{Cs}^{+}$ & $739(2)$ & $1.68(1)$ \\
& $d$-Piv & $\mathrm{Et}_{2} \mathrm{NH}_{2}^{+}$ & $926(10)$ & $1.11(1)$ \\
$\mathbf{2}$ & $d$-Piv & $d$ - $\mathrm{Et}_{2} \mathrm{NH}_{2}^{+}$ & $929(13)$ & $1.08(1)$ \\
$\mathbf{2 b}$ & $d$-Piv & $\mathrm{Cs}^{+}$ & $894(11)$ & $1.07(1)$ \\
$\mathbf{3}$ & $\mathrm{Ad} 1 \mathrm{CO}_{2}$ & ${ }^{n} \mathrm{Pr}_{2} \mathrm{NH}_{2}^{+}$ & $552(4)$ & $0.91(0)$ \\
& $\mathrm{Ad} 1 \mathrm{CO}_{2}$ & $\mathrm{Et}_{2} \mathrm{NH}_{2}^{+}$ & $658(4)$ & $1.07(1)$ \\
$\mathbf{3 b}$ & $\mathrm{Ad} 1 \mathrm{CO}_{2}$ & $\mathrm{Cs}^{+}$ & $898(2)$ & $1.13(0)$ \\
$\mathbf{5}$ & $\mathrm{Ac}$ & ${ }^{n} \mathrm{Pr}_{2} \mathrm{NH}_{2}^{+}$ & $440(4)$ & $1.15(1)$ \\
& $d$-Ac & ${ }^{n} \mathrm{Pr}_{2} \mathrm{NH}_{2}^{+}$ & $538(8)$ & $1.08(1)$ \\
& $\mathrm{Th} 3 \mathrm{CO}{ }_{2}$ & ${ }^{n} \mathrm{Pr}_{2} \mathrm{NH}_{2}^{+}$ & $480(5)$ & $0.97(1)$ \\
& ${ }^{n}$ & ${ }^{+} \mathrm{Pr}_{2} \mathrm{NH}_{2}^{+}$ & $631(2)$ & $1.53(1)$ \\
$\mathbf{6}$ & ${ }^{n} \mathrm{Pr}_{2} \mathrm{NH}_{2}^{+}$ & $340(2)$ & $1.34(1)$ \\
\hline \hline
\end{tabular}

deuteron magnetic moment $\left(\gamma_{\mathrm{H}} / \gamma_{\mathrm{D}} \approx 6.5\right)$ the internuclear dipolar couplings are much weaker and hence the nuclear energy distribution is significantly narrower. The low deuteron magnetic moment leads to weak electronnuclear dipolar coupling making nuclear spin flips less effective in driving dephasing, leading to an increase in $T_{m}$ as reported previously [12].
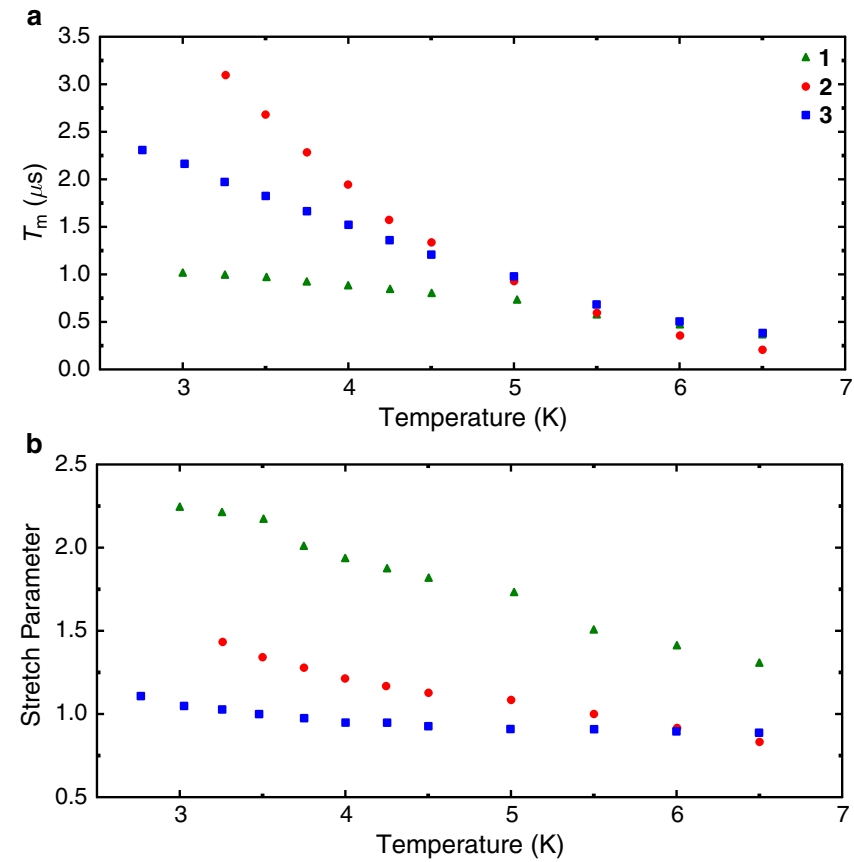

FIG. 2 (color online). Temperature dependence of phase decoherence parameters for representative alkyl-ammonium templated compounds with carboxylate bridging ligands Piv (1), $d$-Piv (2) and $\mathrm{Ad}_{1 C O}(3)$ in frozen $h$-toluene solution.
Making the carboxylate structure more rigid, thereby imposing fixed orientations on the hydrogen atoms which are disordered in the pivalate, leads to compound $\mathbf{3}$. This ordering narrows the nuclear energy distribution, making nuclear spin diffusion less effective. Thus, monoexponential relaxation is observed across almost the entire temperature range with only a slight increase in $x$ at the lowest temperatures. This additional rigidity leads to a doubling of $T_{m}$ for $\mathbf{3}$ over $\mathbf{1}$ at low temperatures, even though the ligands of the former contain 96 more protons in total.

The motions of methyl groups are substantially dependent on their steric hindrance; less sterically hindered methyl groups are more effective in driving relaxation due to spectral diffusion [24]. Whereas rotations of the three methyl groups in each Piv ligand are strongly hindered by their mutual interaction, the methyl groups of each Ac or $\mathrm{EtCO}_{2}$ ligand experience a smaller barrier to rotation. Thus at $5 \mathrm{~K}$, compound 4 exhibits a stretch parameter exceeding 1.5, while compounds 5 and $\mathbf{6}$ (templated around the same cation, but with rotationally freer methyl groups in the bridging ligands) exhibit smaller $x$ and decreased $T_{m}$.

To probe the effect of the nuclei of the central templating cation, we can compare complexes with the same carboxylate ligand, Piv (see Table I). In addition to changes in alkyl chain length it has recently become possible to template $\mathrm{Cr}_{7} \mathrm{Ni}$ around $\mathrm{Cs}^{+} \quad\left(100 \%{ }^{133} \mathrm{Cs}, \quad I=\frac{7}{2}\right.$, $\gamma_{\mathrm{H}} / \gamma_{\mathrm{Cs}} \approx 7.6$ ) [32]. For both Piv and $d$-Piv compounds, there is little dependence on the central templating cation at $5 \mathrm{~K}$ (indicating that the bridging ligand nuclei dominate the relaxation) except in the dimethylammonium $\left(\mathrm{Me}_{2} \mathrm{NH}_{2}^{+}\right)$case for which $T_{m}$ and $x$ are reduced. This trend is reproduced across the $3-7 \mathrm{~K}$ temperature range (data not shown). The anomalous behavior of the 


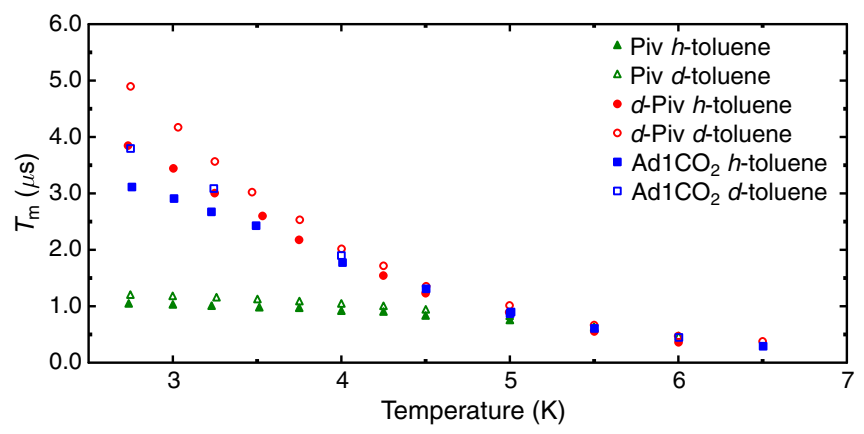

FIG. 3 (color online). Temperature dependence of the phase memory time for three $\mathrm{Cs}^{+}$templated $\mathrm{Cr}_{7} \mathrm{Ni}$ compounds $(\mathbf{1 b}, \mathbf{2 b}$ and $\mathbf{3 b}$ ), in protonated and deuterated frozen toluene solutions.

dimethylammonium cation may relate to a low steric hinderance to methyl rotation within the central pocket of the $\mathrm{Cr}_{7} \mathrm{Ni}$ ring allowing a greater translational diffusion contribution to dephasing.

Variation in the chain length of the alkyl-ammonium cation for the carboxylate ligand $\mathrm{Ad} 1 \mathrm{CO}_{2}$ also produces only a small effect, but in contrast to the Piv compounds there is a marked increase in $T_{m}$ when the templating cation is changed from an alkyl-ammonium to $\mathrm{Cs}^{+}$. This suggests that for this rigid carboxylate bridging ligand, whose protons are inefficient at dephasing, relaxation is dominated by the two methyl groups of the central cation.

The solvent nuclei provide an additional source of dephasing, and though we find that solvent deuteration has little effect on compounds templated around the alkylammonium cation (probably due to the bulk of this template), there is a pronounced increase in $T_{m}$ for compounds templated around the smaller $\mathrm{Cs}^{+}$(see Fig. 3).

The combination of $\mathrm{Cs}^{+}$templated rings with deuterated solvents offers the longest phase memory times. Figure 3 shows that for the $\mathrm{Cs}^{+}$derivatives with the carboxylate bridging ligands $\mathrm{Ad}_{1 \mathrm{CO}}$ and $d$-Piv $T_{m}$ increases with no sign of saturation down to $2.75 \mathrm{~K}$. For the $\mathrm{Ad}_{1 \mathrm{CO}_{2}} \mathrm{com}-$ pound $(3 \mathbf{b})$ at $1.5 \mathrm{~K}$ we find $T_{m}=5.1 \mu \mathrm{s}$ with $x \sim 1.6$, indicating that at sufficiently low temperatures nuclear spin

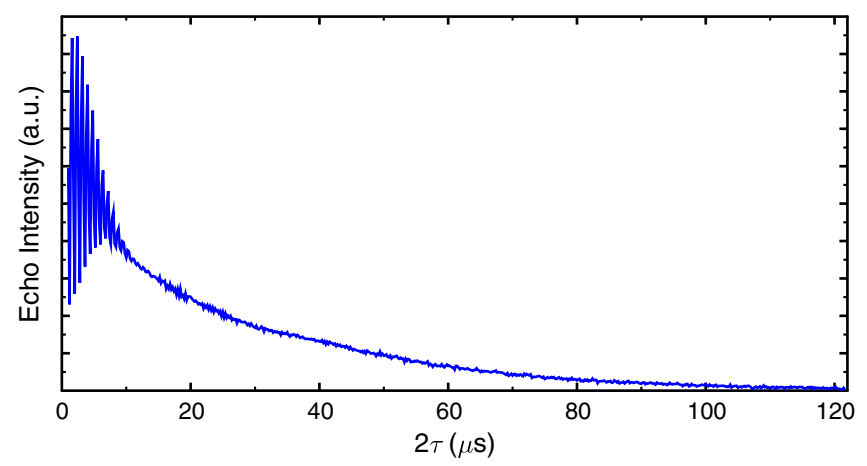

FIG. 4 (color online). Two pulse electron spin-echo decay for $\mathbf{2 b}$ in $d$-toluene at $1.5 \mathrm{~K}$. Electron spin-echo envelope modulations [16] at early time occur at the ${ }^{2} \mathrm{H}$ Larmor frequency. diffusion becomes dominant in this system, as discussed earlier, and this is accompanied by a saturation of $T_{m}$.

As shown in Fig. 4 for the $d$-Piv compound (2b) a more substantial increase in phase memory time is achieved, reaching $T_{m}=15.3 \mu$ s with $x=0.62$ at $1.5 \mathrm{~K}$ with no sign of saturation. A stretch parameter of $x=0.5$ has been derived for the situation in which the motional correlation time is comparable to the Hahn echo duration, but both are shorter than the time scale for nuclear spin diffusion [19].

As with previous studies $[12,33]$ we find no evidence for coupling of the electron spin to the $I=\frac{1}{2}$ fluorine nuclei. The fluoride's high electronegativity ensures that there is little unpaired electron spin density on the fluorine atoms, minimizing exchange coupling between the fluorine nuclei and the electron spin. Dipolar coupling might be expected to offer a decoherence path, but we can conclude from this study that the rate for any such process is low, and certainly less than about $7 \times 10^{4} \mathrm{~s}^{-1}$.

In conclusion, we measured the phase-coherence relaxation time $\left(T_{m}\right)$ in a wide range of structurally related molecular magnets, providing new insights into the dominant dephasing processes in heterometallic $\mathrm{Cr}_{7} M$ molecular magnets, and the many other molecular magnet systems incorporating similar molecular building blocks. This information allows us to increase considerably the phase memory times in these systems, to values exceeding $15 \mu \mathrm{s}$. With coherent manipulations of electron spins possible in a time scale on the order of $10 \mathrm{~ns}$ the single-qubit figure of merit extends to over a thousand. This substantial advance paves the way for attempts to manipulate nontrivial quantum states within coupled or high spin molecular magnet clusters.

We would like to thank J. Harmer, J. J. L. Morton, and O. Rival for useful discussion and R. G. Pritchard and C. A. Muryn for solving the crystal structures. This research was supported by the EPSRC (UK), including for funding of the National EPR Facility in Manchester (EP/D050782/1), the Oxford CAESR facility (EP/D048559/1) and Grants EP/H011714/1 and EP/H012613/1. The project MolSpinQIP acknowledges the financial support of the Future and Emerging Technologies (FET) programme within the Seventh Framework Programme for Research of the European Commission, under FET-Open Grant No. 211284. E. T. S gratefully acknowledges the financial support of the German Research Foundation (DFG). R.E.P.W and A. A. are grateful to the Royal Society for support.

*arzhang.ardavan@ @hysics.ox.ac.uk

[1] D. Gatteschi, R. Sessoli, and J. Villain, Molecular Nanomagnets (Oxford Univeristy Press, Oxford, 2006).

[2] M. N. Leuenberger and D. Loss, Nature (London) 410, 789 (2001).

[3] J. Tejada et al., Nanotechnology 12, 181 (2001). 
[4] G. A. Timco et al., Nature Nanotech. 4, 173 (2009).

[5] G. A. Timco et al., Angew. Chem., Int. Ed. 47, 9681 (2008).

[6] S. Hill et al., Science 302, 1015 (2003).

[7] V. Corradini et al., Phys. Rev. B 79, 14419 (2009).

[8] A. Ghirri et al., Adv. Funct. Mater. 20, 1552 (2010).

[9] F. Meier, J. Levy, and D. Loss, Phys. Rev. Lett. 90, 047901 (2003).

[10] F. Meier, J. Levy, and D. Loss, Phys. Rev. B 68, 134417 (2003).

[11] F. Troiani et al., Phys. Rev. Lett. 94, 190501 (2005).

[12] A. Ardavan et al., Phys. Rev. Lett. 98, 057201 (2007).

[13] N. V. Gerbeleu et al., Dokl. Akad. Nauk SSSR 313, 1459 (1990).

[14] J. van Slageren et al., Chem. Eur. J. 8, 277 (2002).

[15] F. K. Larsen et al., Angew. Chem., Int. Ed. Engl. 42, 101 (2003).

[16] A. Schweiger and G. Jeschke, Principles of Pulsed Electron Paramagnetic Resonance (Oxford University Press, Oxford, 2001).

[17] R. Caciuffo et al., Phys. Rev. B 71, 174407 (2005).

[18] S. Piligkos et al., Chem. Eur. J. 15, 3152 (2009).

[19] S.S. Eaton and G. R. Eaton, in Relaxation Times of Organic Radicals and Transition Metal Ions, edited by G. R. Eaton, S.S. Eaton and L. J. Berliner, Biological Magnetic Resonance Vol. 19 (Springer-Verlag, Berlin, 2000), p. 29.
[20] I. M. Brown, in Time Domain Electron Spin Resonance, edited by L. Kevan and R. N. Schwartz (Wiley Interscience, New York, 1980), Chap. 6.

[21] K. M. Salikhov and Y.D. Tsvetkov, in Time Domain Electron Spin Resonance, edited by L. Kevan and R. N. Schwartz (Wiley Interscience, New York, 1980), Chap. 7.

[22] A. A. Nevzorov and J. H. Freed, J. Chem. Phys. 115, 2416 (2001).

[23] A. A. Nevzorov and J. H. Freed, J. Chem. Phys. 117, 282 (2002).

[24] M. Huber et al., Biophys. Chem. 94, 245 (2001).

[25] S. Saxena and J. H. Freed, J. Phys. Chem. A 101, 7998 (1997).

[26] A. Zecevic et al., Mol. Phys. 95, 1255 (1998).

[27] M. Matsushita, T. Momose, and T. Shida, J. Chem. Phys. 92, 4749 (1990).

[28] S. Kubota et al., Bull. Chem. Soc. Jpn. 68, 140 (1995).

[29] J. H. Freed, J. Chem. Phys. 43, 1710 (1965).

[30] J.M. Hollas, High Resolution Spectroscopy (Wiley, Chichester, 1998), Chap. 5.

[31] J. L. Carolan et al., J. Phys. C 5, 631 (1972).

[32] T. B. Faust et al., Chem. Commun. (Cambridge) 46, 6258 (2010).

[33] C. Schlegel et al., Phys. Rev. B 83, 134407 (2011).

[34] See Supplemental Material at http://link.aps.org/ supplemental/10.1103/PhysRevLett.108.107204 for details of sample preparation, electron spin resonance measurements and analysis, crystallographic data and the abundance of hydrogen atoms in the molecular building blocks. 